
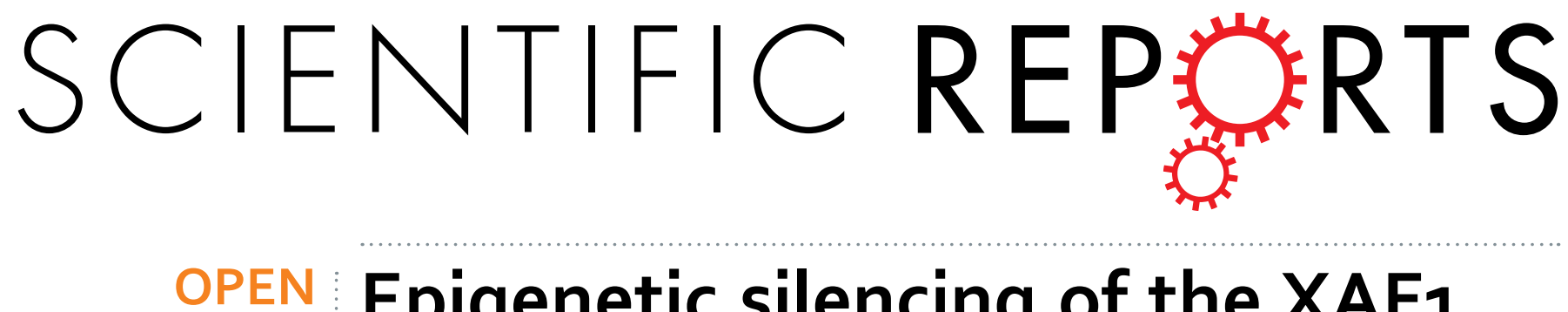

\title{
Epigenetic silencing of the XAF1 gene is mediated by the loss of CTCF binding
}

Received: 20 May 2015

Accepted: 07 September 2015

Published: 07 October 2015

\author{
Georgina Victoria-Acosta ${ }^{1}$, Karla Vazquez-Santillan ${ }^{2}$, Luis Jimenez-Hernandez ${ }^{2}$, \\ Laura Muñoz-Galindo ${ }^{2}$, Vilma Maldonado ${ }^{2}$, Gustavo Ulises Martinez-Ruiz ${ }^{1,3}$ \& \\ Jorge Melendez-Zajgla ${ }^{1}$
}

$\mathrm{XAF}_{1}$ is a tumour suppressor gene that compromises cell viability by modulating different cellular events such as mitosis, cell cycle progression and apoptosis. In cancer, the XAF1 gene is commonly silenced by CpG-dinucleotide hypermethylation of its promoter. DNA demethylating agents induce transcriptional reactivation of $\mathrm{XAF}_{1}$, sensitizing cancer cells to therapy. The molecular mechanisms that mediate promoter CpG methylation have not been previously studied. Here, we demonstrate that CTCF interacts with the $X A F_{1}$ promoter in vivo in a methylation-sensitive manner. By transgene assays, we demonstrate that CTCF mediates the open-chromatin configuration of the XAF1 promoter, inhibiting both $\mathrm{CPG}$-dinucleotide methylation and repressive histone posttranslational modifications. In addition, the absence of CTCF in the $X A F_{1}$ promoter inhibits transcriptional activation induced by well-known apoptosis activators. We report for the first time that epigenetic silencing of the $X A F_{1}$ gene is a consequence of the loss of CTCF binding.

The tumour-suppressor gene X-linked inhibitor of the apoptosis (XIAP)-associated factor 1 (XAF) favours apoptosis by inhibiting XIAP ${ }^{1-5}$, which is one of the most important members of the inhibitors of apoptosis protein (IAP) family. In addition, XAF1 also presents XIAP-independent proapoptotic actions that contribute to its tumour suppressor gene activity ${ }^{6-8}$. XAF1 expression is absent or decreased in gastric $^{9}$, ovarian ${ }^{10}$, pancreatic $^{11}$, esophageal ${ }^{12}$, colon $^{9}$, hepatic ${ }^{13}$, melanoma ${ }^{14}$ and urogenital tumours ${ }^{15-17}$. Although loss of heterozygosity has been showed to be associated to XAF1 expression absence ${ }^{18}$, promoter CpG dinucleotide hypermethylation appears to be the principal cause of altered XAF1 expression $^{9,12,17}$. Exposure to demethylating agents such as 5-azacytidine readily induces the reestablishment of XAF1 expression, thereby increasing the sensitivity to drug-induced apoptosis ${ }^{12,19,20}$. In xenograft models, ectopic XAF1 expression impedes tumour formation and prolongs the survival of tumour-bearing mice $^{21,22}$. However, the molecular mediators of the hypermethylated state and decreased expression are currently unknown.

CTCF is a multitask protein involved in gene regulation. This protein functions as a transcriptional regulator, enhancer blocker and chromatin barrier ${ }^{23}$. These actions are secondary to its main function as a genome-wide organizer of chromatin architecture ${ }^{24,25}$. The biological actions of CTCF are explained by its ability to function as a DNA-binding protein scaffold. CTCF interacts with its DNA-binding sites in a methylation-sensitive fashion, thereby impeding the methylation of imprinting control regions ${ }^{26,27}$. In cancer, it has been described that CTCF is able to modulate the histone posttranslational modification (HPM) status and CpG methylation from several tumour suppressor genes ${ }^{28}$.

${ }^{1}$ Functional Cancer Genomics Laboratory, National Institute of Genomic Medicine, Mexico D.F., 14610, Mexico. ${ }^{2}$ Epigenetics Laboratory, National Institute of Genomic Medicine, Mexico D.F., 14610, México. 3Unit of Investigative Research on Oncological Disease, Children's Hospital of Mexico "Federico Gomez", Mexico City, Mexico. Correspondence and requests for materials should be addressed to G.U.M.-R. (email: gustavobambam@ gmail.com) or J.M.-Z. (email: jmelendez@inmegen.gob.mx) 
a

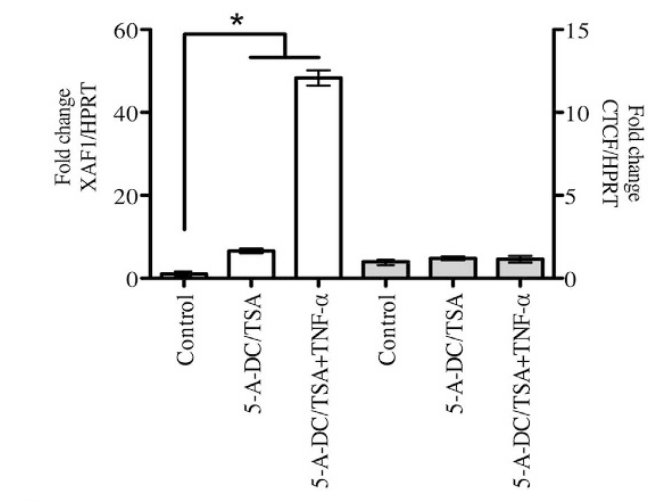

C

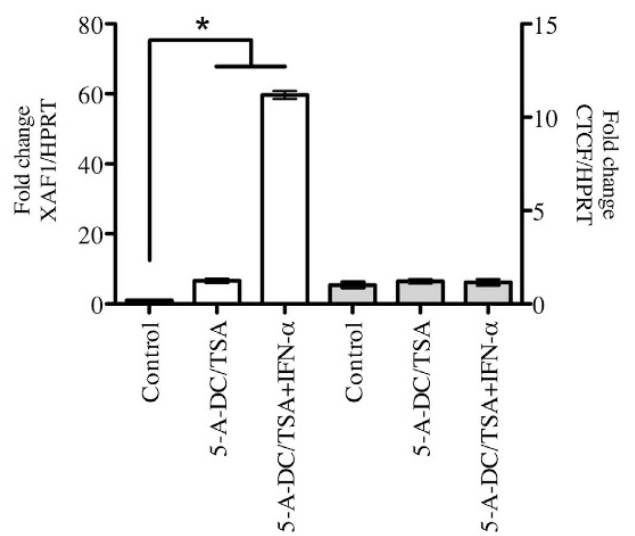

b

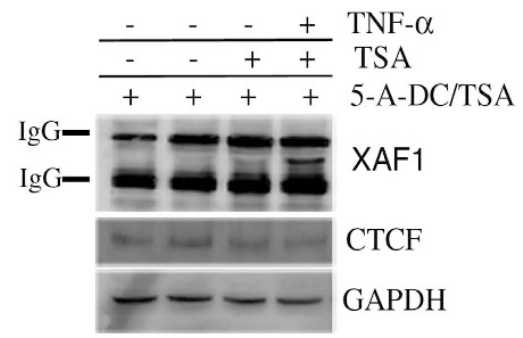

d

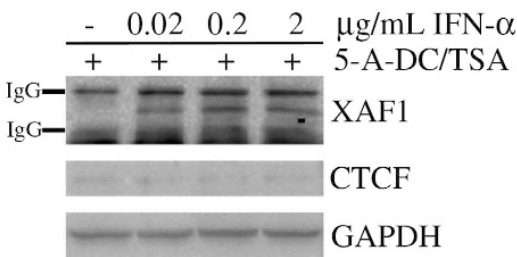

Figure 1. XAF1 expression is induced by either TNF- $\alpha$ or IFN- $\alpha$ in demethylating conditions. (a) MCF-7 cells were pre-treated with 5-Aza-2'-deoxycytidine $(5 \mu \mathrm{M})$ and Trichostatin-A $(0.2 \mu \mathrm{M})$ for 3 days before stimulation with TNF- $\alpha(20 \mathrm{ng} / \mathrm{mL})$ for $24 \mathrm{~h}$. Quantitative PCR (qPCR) analysis of XAF1 and CTCF mRNA expression was performed. HPRT mRNA was used as loading control. Results are presented in terms of fold change. The means from three independent experiments were plotted with $\pm \mathrm{SEM},{ }^{\star} P<0.05$. (b) MCF-7 cells were treated as shown in (a). Using a specific antibody, XAF1 was immunoprecipitated from equal quantities of total extracted proteins for each condition. XAF1, CTCF and GAPDH protein levels were measured by Western blot. (c) MCF-7 cells were pre-treated as in (a) before stimulation with IFN- $\alpha$ at the indicated concentrations. mRNA expression of both XAF1 and CTCF was analysed by qPCR after normalizing with HPRT mRNA. The mean from three independent replicates were plotted with $+\mathrm{SEM}$, ${ }^{\star} P<0.05$. (d) MCF-7 cells were pre-treated and stimulated as shown in (c). Western blot analysis was performed as shown in (b). 5-Aza-2'-deoxycytidine (5-A-DC); Trichostatin-A (TSA).

Here, we demonstrated that CTCF directly regulates XAF1 expression by binding to a methylation-sensitive CTCF-binding site in its promoter. The absence of CTCF promotes epigenetic silencing of the XAF1 promoter by both accelerated CpG-dinucleotide methylation and the transition from active to repressive HPMs. Importantly, in cancer cell lines, the lack of CTCF regulation on the $X A F 1$ promoter via methylation on its cognate binding site partially blocks its transcriptional responsiveness to two well-known transcriptional activators, TNF- $\alpha$ or IFN- $\alpha$. These findings uncover for the first time an epigenetic mechanism involved in establishing the repressive configuration of the XAF1 promoter and, consequently, transcriptional unresponsiveness.

\section{Results}

Specific CpG-dinucleotide methylation impedes full XAF1 responsiveness to either TNF- $\alpha$ or IFN- $\alpha$ in MCF-7 cells. As expected based on previous reports showing that XAF1 promoter is hypermethylated in cancer ${ }^{9,12,17}$, here, pre-exposure to demethylating agents increased the transcriptional activation of XAF1 in basal conditions (Supplementary Fig.1a). To test XAF1 dynamic expression, we used two well-known XAF1 transcriptional activators, TNF- $\alpha$ and IFN- $\alpha^{29-31}$ Demethylating conditions were required to display full transcriptional activation of XAF1 at both the mRNA and protein levels 
a

b
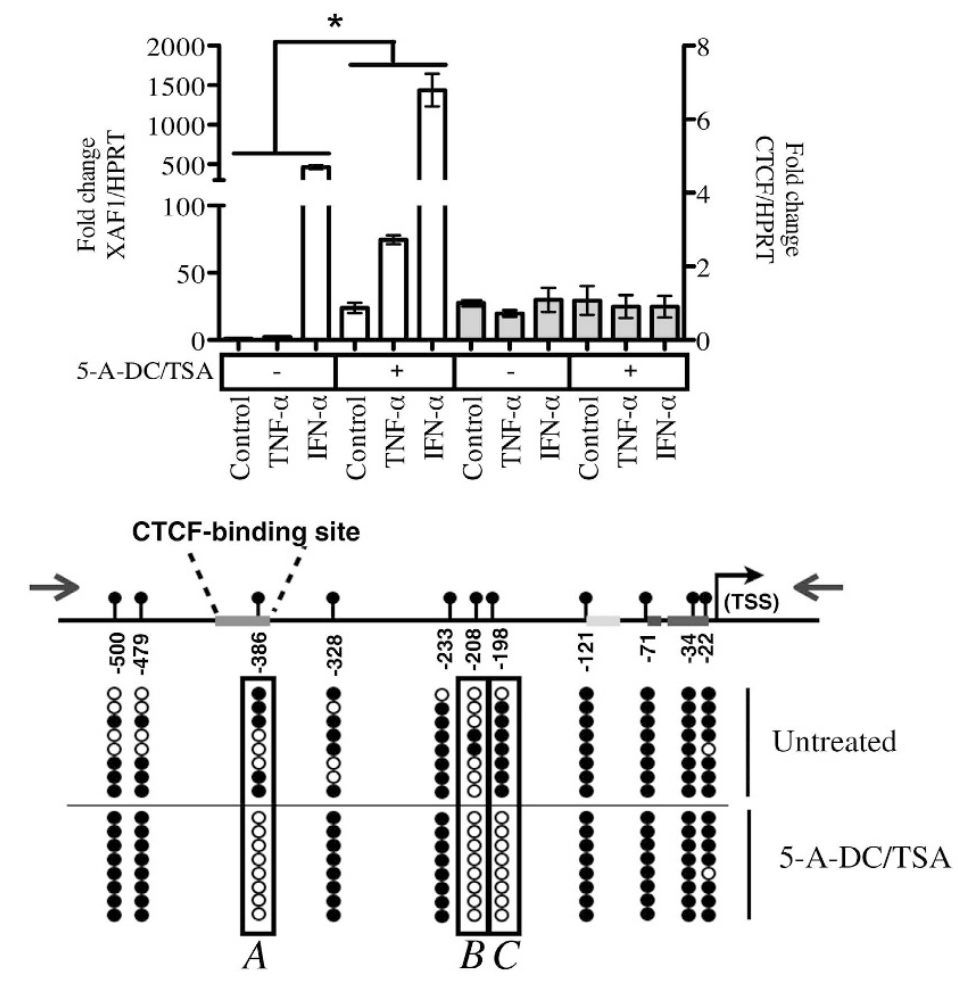

C

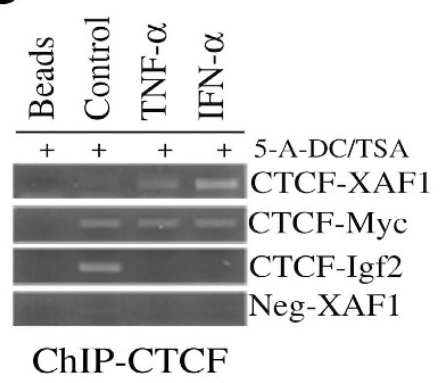

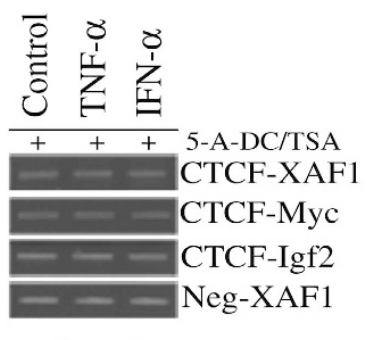

Input

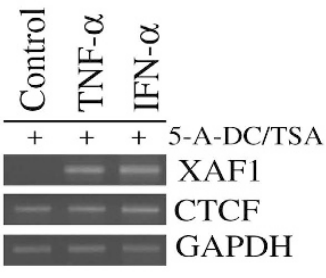

RT-PCR

Figure 2. CTCF interacts with the XAF1 promoter. (a) ACHN cells were pre-treated with 5-Aza$2^{\prime}$-deoxycytidine $(5 \mu \mathrm{M})$ and Trichostatin-A $(0.2 \mu \mathrm{M})$ for 3 days before stimulation with either TNF- $\alpha$ or IFN- $\alpha$. Non-demethylated cells were stimulated with either TNF- $\alpha$ or IFN- $\alpha$. mRNA expression of XAF1, $C T C F$ and HPRT was analysed by qPCR. Results are presented in terms of fold change. The means from three independent experiments were plotted with \pm SEM, ${ }^{\star} P<0.05$. (b) MCF-7 cells were either treated or not treated with demethylating agents, as shown in (a). Bisulphite sequencing was then performed. A schematic representation of the XAF1 promoter shows the locations of $11 \mathrm{CpG}$-dinucleotides sites from -22 to $-500 \mathrm{bp}$ relative to the TSS. Methylated and unmethylated CpGs are depicted as filled and open circles, respectively (c) MCF-7 cells were treated as shown in (a). ChIP assays were performed using a specific antibody against CTCF protein. The CTCF-binding site in the XAF1 promoter was analysed by PCR in the DNA recovered after ChIP (Left panel). As positive and negative controls of CTCF-DNA interaction, three specific sets of primers were included. Two of them were directed to previously validated CTCF binding sites (c-Myc and IGF2) as positive controls, and one was a negative control. The input represents soluble chromatin that was reversed cross-linked and amplified by PCR (central panel). RT-PCR was performed from cells used for ChIP assays. 5-Aza-2'-deoxycytidine (5-A-DC); Trichostatin-A (TSA).

after TNF- $\alpha$ (Fig. 1a,b) or IFN- $\alpha$ (Fig. 1c,d) exposure. To extend these observations to another unrelated cancer cell line, we used ACHN cells, which have previously been shown to be responsive to IFN- $\alpha$ in demethylating conditions ${ }^{32}$. As observed with MCF-7 cells, we observed a dramatic increase in XAF1 responsiveness in demethylating conditions (Fig. 2a). As a positive control, we used the Colo205 cell line that presents an unmethylated XAF1 promoter ${ }^{9}$. Even without previous exposure to epigenetic modifiers, we observed a clear XAF1 transcriptional activation by TNF- $\alpha$ exposure (Supplementary Fig. 1b). We then reasoned that differential dinucleotide CpG methylation between control cells and cells treated with demethylating agents could help us to identify which DNA segments are important for the full responsiveness of XAF1. To this end, we performed bisulphite genomic sequencing using a specific set of 
a

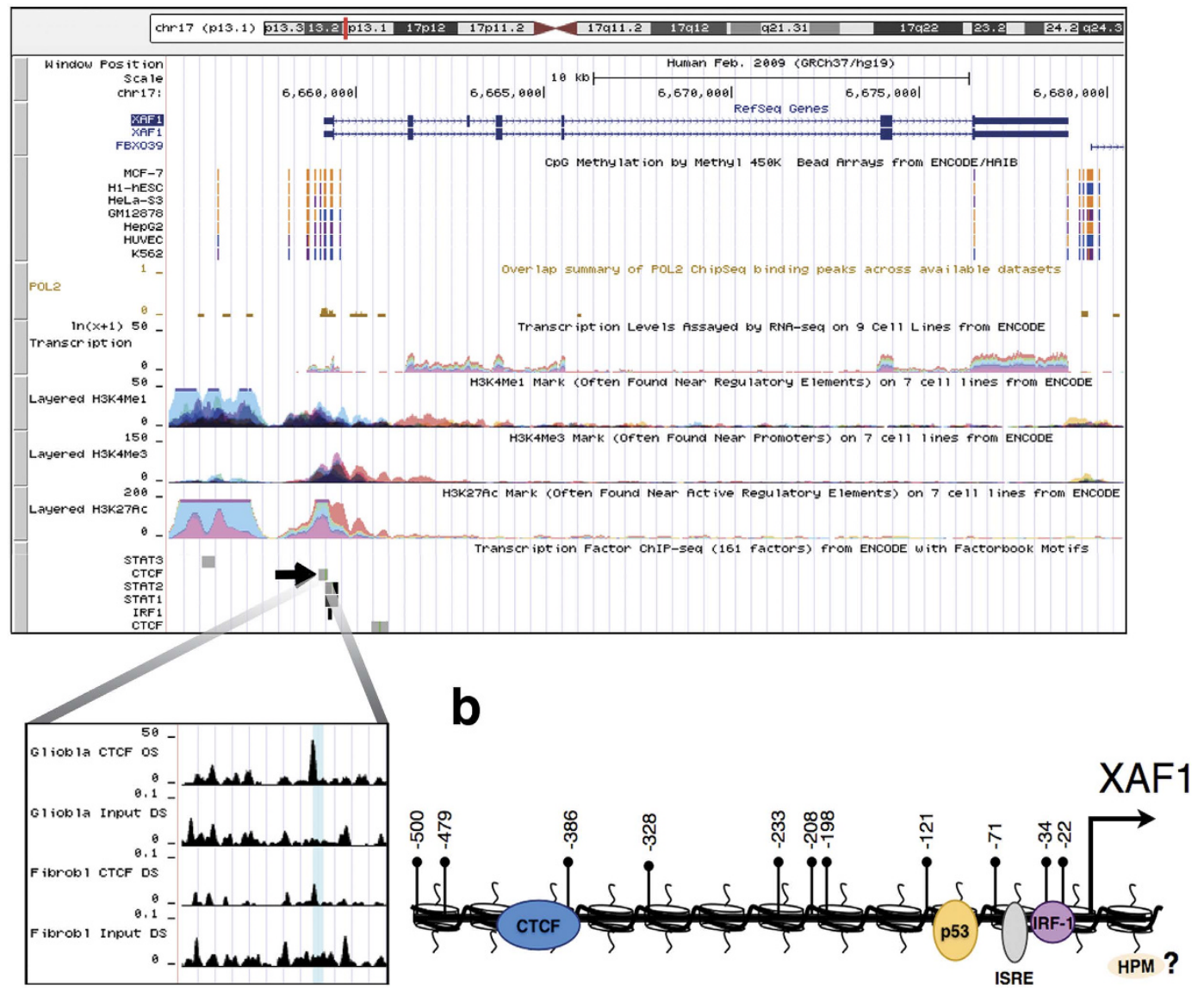

Figure 3. Features of the XAF1 promoter. (a) The XAF1 promoter visualized in the UCSC genome browser. The picture illustrates the CpG-methylation status from different types of cell lines. Additionally, the profiles of several histone posttranslational modifications such as H3K4Me1, H3K4Me3 and H3K27Ac are presented from different cell lines. Several transcription factor binding sites obtained from ChIP-Seq data are also shown. At a higher resolution, the CTCF binding site in the XAF1 promoter in glioblastoma and fibroblast cells is shown. (b) Schematic representation of the XAF1 promoter showing the CpG-dinucleotide positions from -22 to $-500 \mathrm{bp}$ relative to the transcription start site and the previously described binding sites for IRF-1, ISRE, p53 and the uncharacterized CTCF binding site. Histone posttranslational modification (HPM).

primers to amplify the $X A F 1$ promoter. Exposure to 5-aza-2'-deoxycytidine (5-A-DC) and trichostatin-A (TSA) induced consistent demethylation of three CpG dinucleotides in MCF-7 cells (Fig. 2b; A, B and C). These results indicate that full transcriptional activation of the XAF1 gene is associated with a specific CpG-dinucleotide methylation state of its promoter.

CTCF interacts with the $X A F_{1}$ promoter when cells are stimulated with TNF- $\alpha$ or IFN- $\alpha$. CTCF is known to regulate the expression of diverse tumour suppressor genes by directly binding to promoter sequences $^{28}$. We searched for transcription binding sites in a window of $-/+10 \mathrm{bp}$ of the DNA sequence adjacent to each $\mathrm{CpG}$ that was demethylated as a consequence of epigenetic modifiers. Interestingly, we identify a putative CTCF binding site that overlapped the CpG dinucleotide located at -388 bp relative to the transcription start site (Fig. 2b). Supporting the relevance of this site, its presence was confirmed in an experimentally validated CTCF-binding site database ${ }^{33}$ (Fig. 3a,b). To experimentally validate this, ChIP assays were performed in MCF-7 cells after stimulation with TNF- $\alpha$ or IFN- $\alpha$. As shown in Fig. 2c, in basal conditions, we could not find a detectable association of CTCF with the putative CTCF binding site in the XAF1 promoter. This result could be explained by a methylation-sensitive CTCF binding mechanism. To directly test this, we exposed the cells to demethylating agents before stimulation with TNF- $\alpha$ or IFN- $\alpha$. As expected, the association of CTCF with the XAF1 promoter was detected only after DNA demethylation and stimulation with TNF- $\alpha$ or IFN- $\alpha$ (Fig. $2 \mathrm{c}$ ). This observation correlated with an increased transcriptional activation when the cells were previously exposed to the epigenetic modifiers (Fig. 2c, third panel). Additionally, we validated this CTCF binding site using an additional 
a

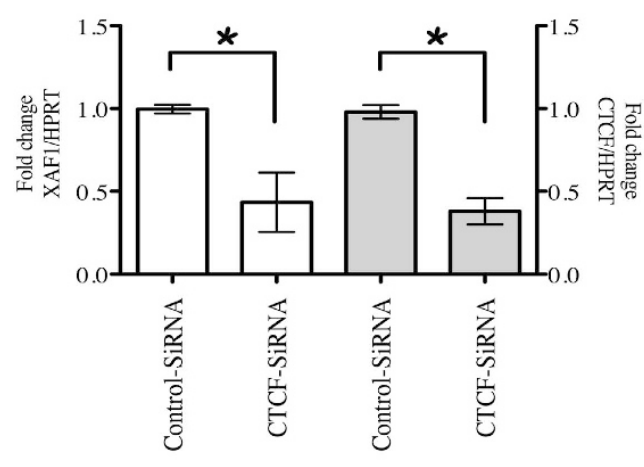

C

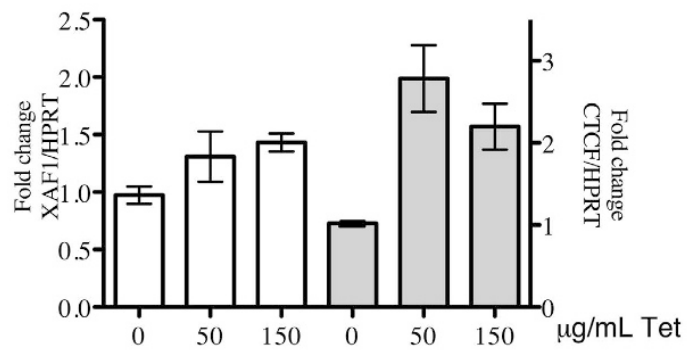

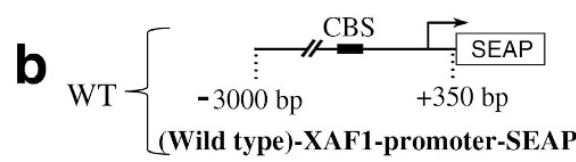
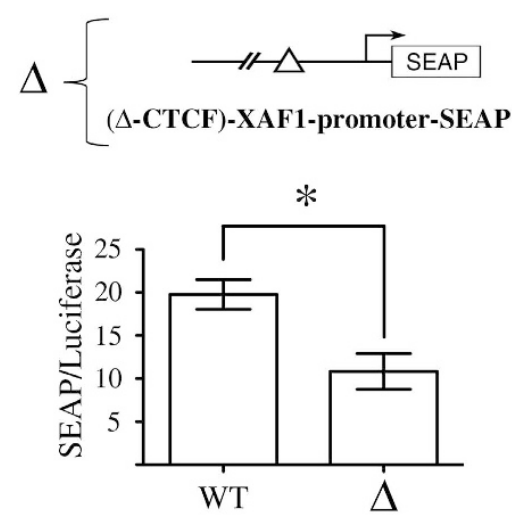

d

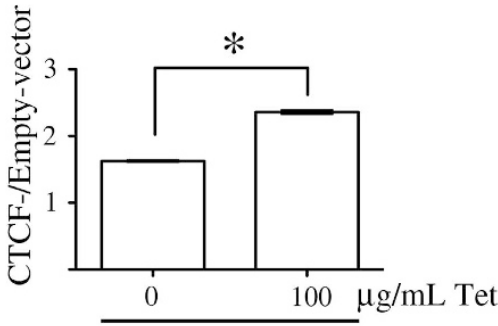

(Wild type)-XAF1-promoter-SEAP

Figure 4. CTCF regulates transcriptional activation of the XAF1 gene. (a) ACHN cells were pre-treated with 5-aza- $2^{\prime}$-deoxycytidine $(5 \mu \mathrm{M})$ and Trichostatin-A $(0.2 \mu \mathrm{M})$ for 3 days. After that, the cells were transiently transfected with CTCF siRNAs or control scramble siRNAs. qPCR analyses were performed to measure the expression of both XAF1 and CTCF mRNA. HPRT was used as loading control. The means from three independent experiments were plotted with $+\mathrm{SEM},{ }^{\star} P<0.05$. (b) MCF-7 cells were transitorily co-transfected with both Wild-type-XAF1-promoter-SEAP or $\triangle$-CTCF-XAF1-promoter-SEAP constructs and pMetLuc, which was used for transfection normalization. Data are represented as the means + SEM from three independent experiments, ${ }^{\star} P<0.05$. (c) MCF-7 stable clones of CTCF/Tet-On were stimulated with tetracycline at the indicated concentrations. Using qPCR assays, XAF1 and CTCF mRNA expression was normalized to HPRT, used as a loading control. The mean and range were plotted from two independent stable cell lines. (d) MCF-7-CTCF/Tet-On and MCF-7 Empty/Tet-On cell lines were transitory co-transfected with Wild-type-XAF1-promoter-SEAP and pMetLuc. After $48 \mathrm{~h}$, tetracycline was added for $24 \mathrm{~h}$. Data are represented as the means + SEM from three independent experiments, ${ }^{\star} P<0.05$. 5-Aza-2'-deoxycytidine (5-A-DC); Trichostatin-A (TSA); CTCF-binding site (CBS).

cell line. As in MCF-7 cells, we observed a dramatic increase in the interaction of CTCF with the XAF1 promoter when the cells were stimulated with either TNF- $\alpha$ or IFN- $\alpha$ after exposure to demethylating agents (Supplementary Fig. 1c). These results support a methylation-sensitive association of CTCF with the $X A F 1$ promoter.

XAF1 expression is regulated by CTCF. To further define the role of CTCF on XAF1 mRNA expression, we used specific siRNAs to downregulate CTCF expression in a series of loss-of-function experiments. We verified the efficacy of these siRNAs at both mRNA and protein levels (Supplementary Fig. 1d). Because previous reports have shown that demethylating agents increase XAF1 induction by IFN in ACHN cells ${ }^{32}$, we used this cell line to analyse the effect of these siRNAs on XAF1 transcriptional responsiveness to TNF- $\alpha$ or IFN- $\alpha$. As described above, demethylating conditions are necessary to uncover the CTCF-binding site (Fig. 2b,c). We clearly observed lower levels of XAF1 mRNA in cells transfected with the siRNAs against CTCF than those transfected with control siRNAs (Fig. 4a). Additionally, we confirmed the regulatory effect of CTCF on the XAF1 promoter using the secreted alkaline phosphatase (SEAP) reporter gene assays. In these assays, the enzymatic activity drove by the $X A F 1$ promoter region comprising $-3000 \mathrm{bp}$ to $+350 \mathrm{bp}$ relative to the transcription 
start site (Wild-type-XAF1-promoter-SEAP) was compared with the same region with a deletion of the core CTCF binding site ( $\triangle$-CTCF-XAF1-promoter-SEAP). The absence of the CTCF binding site in the XAF1 promoter inhibits its basal transcriptional activation (Fig. 4b). To further support these results, we also conducted gain-of-function experiments by analysing the effects of CTCF overexpression on XAF1 mRNA expression. To achieve this, we engineered a Tet-on CTCF system in the MCF-7 cell line. In demethylating conditions, the overexpression of CTCF mediated by tetracycline addition induced transcriptional activation of XAF1 (Fig. 4c). Additionally, these cells were transfected with the Wild-type-XAF1-promoter-SEAP construct. After tetracycline addition, we observed a significant increase in the enzymatic activity of the reporter in cells overexpressing CTCF (Fig. 4d). On the other hand, we evaluated the role of CTCF over-expression in terms of transcriptional responsiveness of XAF1 in TNF- $\alpha$ - or IFN- $\alpha$-treated cells. Although we detected an increase in XAF1 levels in cells over-expressing CTCF, the TNF- $\alpha$ or IFN- $\alpha$-mediated transcriptional increase was not modified by CTCF overexpression (supplementary Fig. 2a). This points toward a shared signalling mechanism and supports the role of CTCF in the effects of these cytokines on XAF1 regulation, with additional factors needed for maximal responsiveness. Thus, both gain and loss of function approaches showed the participation of CTCF in XAF1 expression.

CTCF protects the XAF1 gene from epigenetic silencing. The insulating action of CTCF protects several genes from epigenetic silencing ${ }^{28,34}$. In particular, it has been described that the absence of CTCF in tumour suppressor gene promoters induces their epigenetic silencing, which supports the role of CTCF in cancer ${ }^{35-37}$. To test the possible epigenetic-mediated regulation of CTCF on the XAF1 gene, we compared the $X A F 1$ promoter activity in a genomic integrated context by measuring a GFP reporter gene. For this, we compared the wild type XAF1 promoter with the CTCF-deletion ( $\triangle$-CTCF-XAF1) construct. Supporting the insulating role of CTCF on the XAF1 gene, cells with the $\triangle$-CTCF-XAF1 promoter showed lower GFP levels than those with the wild-type XAF1 promoter after 60 days of continuous culture (Fig. 5a). To further support this finding, single-cell clones for each transfection were isolated and propagated for an additional 35 days. As expected by the previous result, silencing of GFP expression levels was mainly observed in single-cell clones with the integrated $\Delta$-CTCF-XAF1-promoter (Fig. 5a), pointing toward an epigenetic-protective effect of the CTCF binding site. A possible alternative explanation for the difference in GFP expression levels between transfections could be attributed to a distinct number of integration events. To exclude this possibility, the transgene copy number was measured by real-time PCR as previously reported ${ }^{38}$. The difference between GFP expression levels driven by the $\triangle$-CTCF-XAF1-promoter and wild-type-XAF1-promoter was independent of the transgene copy number (Supplementary Fig. 2b). To gain insight into the epigenetic mechanism involved in GFP silencing of the $\triangle$-CTCF-XAF1-promoter, we hypothesized that loss of the CTCF-binding site could promote 1) accelerated $\mathrm{CpG}$ methylation or 2) acquisition of a repressive chromatin configuration based on HPMs (or both). To test the first hypothesis, sequencing of the sodium bisulphite-modified genomic DNA from three single-cell clones for each transfection was performed. To discriminate endogenous XAF1 promoter amplification, a nested-PCR strategy was performed in which the first set of primers annealed to plasmid sequences surrounding the exogenous $X A F 1$ promoter (Fig. $5 \mathrm{~b}$ and Supplementary Fig. 2c). We observed that the $\triangle$-CTCF-XAF1-promoter is more susceptible to dinucleotide-CpG methylation than the wild-type-XAF1-promoter in a genomic-integrated context (Fig. 5b). To test our second hypothesis, ChIP assays were performed using specific antibodies directed to $\mathrm{H} 3 \mathrm{~K} 4-2$ me or $\mathrm{H} 3 \mathrm{~K} 9-3$ me posttranslational modifications in single-cell clones from each stable transfection. To interrogate the relative enrichment of repressive or active HPMs in the transgene, we designed a pair of primers that anneal to the plasmid sequence immediately after the exogenous XAF1 promoter (Fig. 5c and Supplementary Fig. 2c). Lower levels of the $\mathrm{H} 3 \mathrm{~K} 4-2$ me posttranslational modification, a marker for transcription activity, were observed in cell single clones from the $\Delta$-CTCF XAF1-promoter (Fig. 5c). As expected, the $\Delta-C T C F$ XAF1 promoter was enriched with the repressive H3K9-3me modification (Fig. 5c). Overall, these findings support the notion that CTCF regulates DNA methylation in the XAF1 promoter; thus, loss of CTCF in its cognate-binding site induces DNA-methylation and polarization from active to repressive HPM, which in turn induces transcriptional repression.

XAF1 expression is modulated by CTCF in apoptotic conditions. It has been well described that XAF1 expression reactivation has a crucial role in apoptosis induced by TNF- $\alpha /$ cycloheximide (CHX) or IFN- $\alpha /$ TNF-related apoptosis-inducing ligand (TRAIL) ${ }^{6,22}$. To test if CTCF could regulate XAF1 expression in apoptotic conditions, MCF-7 cells were exposed to either TNF- $\alpha / C H X$ or IFN- $\alpha /$ TRAIL. Cytotoxicity induced by the co-treatment of either TNF- $\alpha / \mathrm{CHX}$ or IFN- $\alpha /$ TRAIL was analysed by cell viability assays (Supplementary Fig. 2d). As expected, we observed the transcriptional activation of XAF1 after exposure to both regimens (Fig. 6a,b). To assess the biological relevance of CTCF-mediated XAF1 transcription, single-cell clones with the wild type- or $\triangle$-CTCF-XAF1 promoter were exposed to inducers of apoptosis. After that, FACS was used to measure GFP-reporter gene activity. Whereas the wild-type promoter activity correlated with the XAF1 transcriptional activation, the $\Delta$-CTCF-XAF1 promoter did not present any transcriptional activity (Fig. 6c). Several reports have shown that XAF1 is an IFN-stimulated gene in cancer cells ${ }^{30,39,40}$. Because its promoter is commonly hypermethylated in these 
a
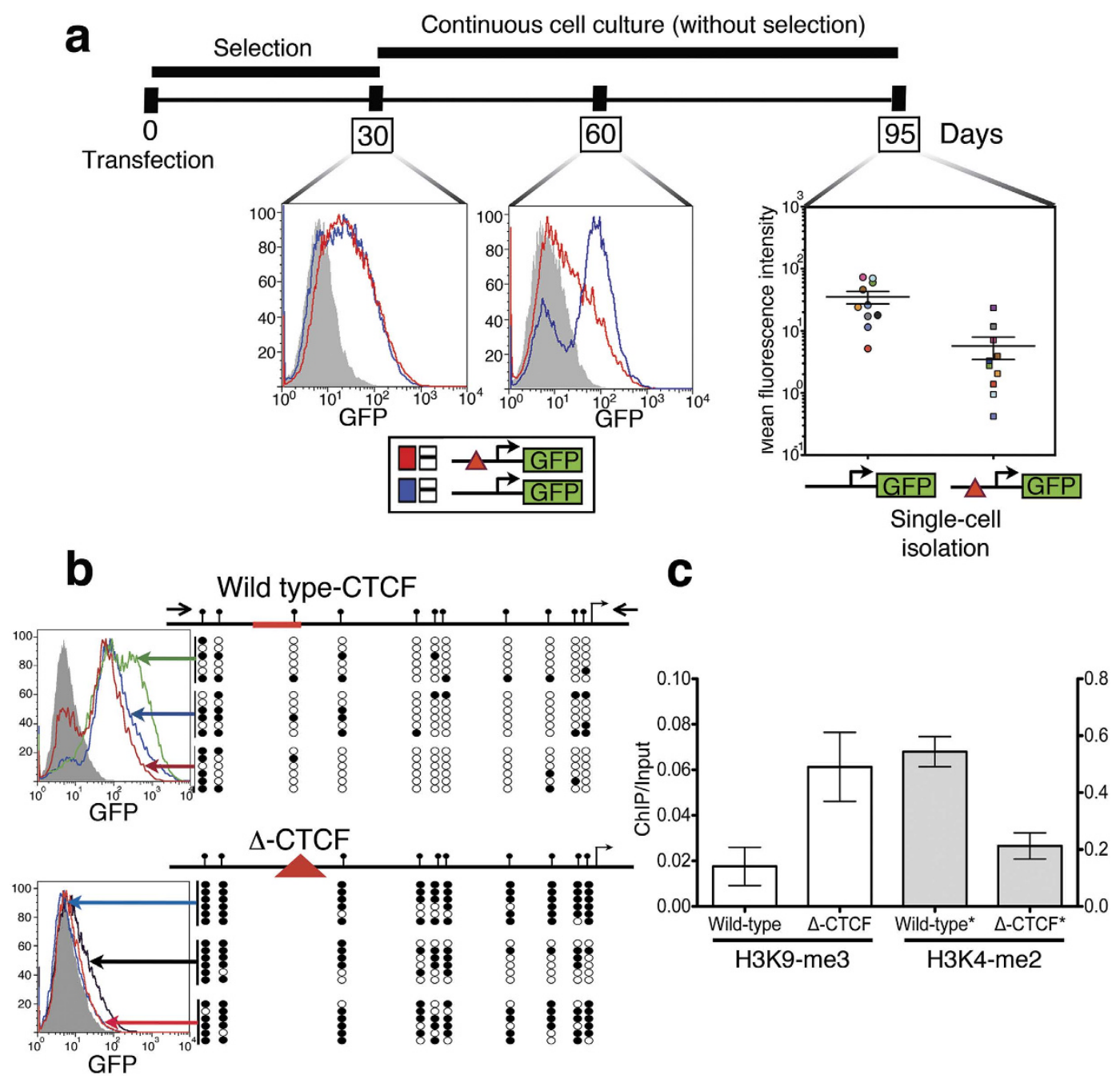

Figure 5. CTCF maintains an open-chromatin configuration in the $X A F 1$ promoter in transgene assays. (a) The timeline for stable transgenic cell line generation using peGFPN1-XAF1 or peGFPN1- $\Delta-\mathrm{CTCF}$ XAF1 plasmids. The detection of GFP expression for each cell line was performed using FACS. Single-cell clones were generated at day 60. (b) Right, bisulphite sequencing was performed from single-cell clones containing either peGFPN1-XAF1 or peGFPN1- $\triangle$-CTCF-XAF1 constructs. The exogenous XAF1 promoter was specifically amplified using a nested-PCR strategy in which the first amplification was performed using primers recognizing plasmid sequences. Methylated and unmethylated CpGs are depicted as filled and open circles, respectively. Left, histograms from each single cell clones are showed (c) ChIP assays were performed from stable single-cell clones using specific antibodies against H3K4-2me or H3K9-3me. Data are represented as the means \pm SEM from three single-cell clones. Red triangle symbols the deletion of the CTCF binding site.

cells, transcriptional activation of the XAF1 gene could be dependent on IFN- $\alpha$-mediated demethylation and could thus rely on $\mathrm{CTCF}^{20}$. Supporting this hypothesis, we found that single-cell clones with the $\triangle$-CTCF-XAF1 promoter were unable to respond to IFN- $\alpha$, indicating that CTCF could be relevant in the IFN- $\alpha$-mediated induction of XAF1 (Fig. $6 \mathrm{c}$ ).

\section{Discussion}

In cancer, it has been described that the $X A F 1$ gene is transcriptionally silenced by CpG-dinucleotide hypermethylation in its promoter ${ }^{9,17,41}$. Exposure to demethylating agents induces XAF1 transcriptional activation, thereby compromising cell viability by promoting apoptosis ${ }^{4,21}$, mitotic catastrophe $e^{4,8}$ or cell cycle inhibition $^{8}$. Thus, CpG methylation in the XAF1 promoter represents the main epigenetic mechanism involved in XAF1 silencing and, consequently, in resistance against apoptosis. However, the deregulation of epigenetic mechanism is implicated in a variety of diseases ${ }^{42}$, including cancer ${ }^{43-45}$. CTCF is a multi-task protein involved in chromatin regulation, with profound consequences in gene expres$\operatorname{sion}^{23,24}$. In a panel of breast cancer cell lines, heightened CTCF expression was associated with apoptosis 
a

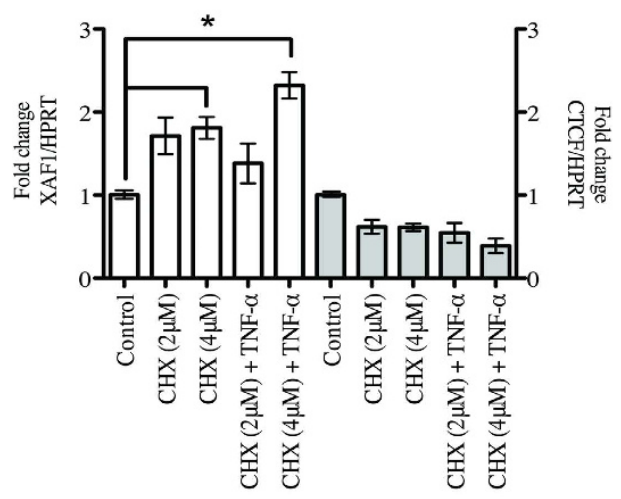

b

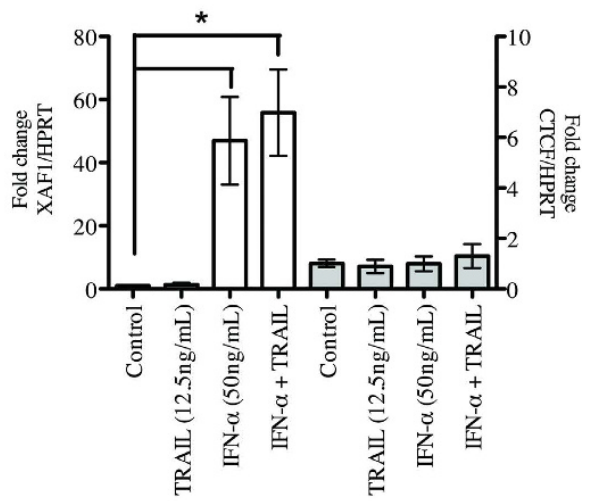

C

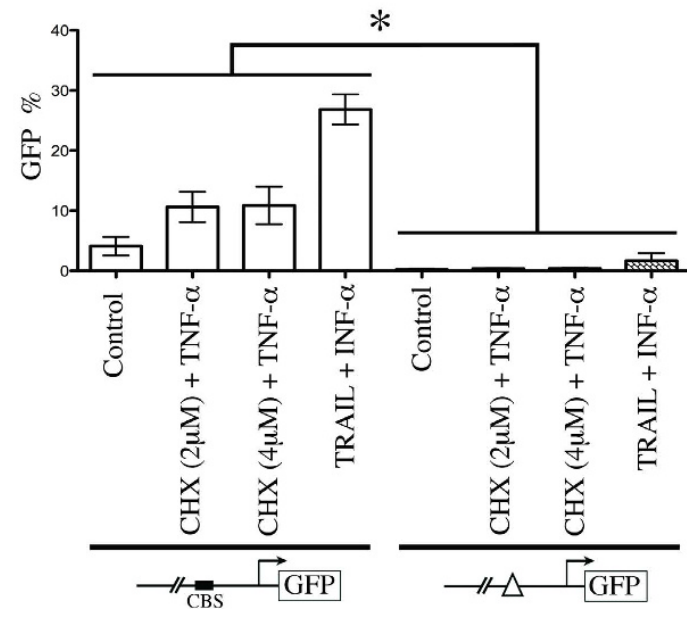

Figure 6. The CTCF binding site in the XAF1 promoter mediates XAF1 responsiveness to activators in apoptotic conditions. (a) MCF-7 cells were treated with 5-Aza- $2^{\prime}$-deoxycytidine $(5 \mu \mathrm{M})$ and Trichostatin-A $(0.2 \mu \mathrm{M})$ for 3 days before stimulation with TNF- $\alpha$ in the presence of cycloheximide (TNF- $\alpha+$ CHX) (left panel). qPCR analyses of XAF1 and CTCF mRNA expression were performed. HPRT mRNA was used as loading control. Results are presented as fold change. Data are represented as the means $\pm \mathrm{SEM}$ from three independent experiments, ${ }^{\star} P<0.05$. (b) MCF-7 cells were pre-treated as shown in (A) before the addition of IFN- $\alpha$ in the presence of TRAIL (IFN- $\alpha+$ TRAIL) (right panel). The expression of XAF1 and CTCF and HPRT was determined by qPCR. HPRT was used as loading control. (c) Stable single-cell clones containing either peGFPN1-XAF1 or peGFPN1- $\Delta$-CTCF-XAF1 constructs were stimulated with either TNF- $\alpha+$ CHX or IFN- $\alpha+$ TRAIL. After, GFP protein levels were measured using FACS. Data are represented as the mean $\mathrm{SD}$ of four single-cell clones from each transfection, ${ }^{\star} P<0.05$.

resistance ${ }^{46}$. The protective action of CTCF is explainable, in part, by negative regulation of the Bax gene, which increases the apoptotic threshold ${ }^{47}$. It has also been clearly demonstrated that CTCF regulates the chromatin configuration of many tumour suppressor genes, affecting their transcription rates ${ }^{28}$. Here, we describe that CTCF interacts with the XAF1 promoter, thereby regulating its chromatin configuration and, consequently, its transcriptional responsiveness to well-known activators. We were able to demonstrate two biological scenarios. First, CTCF maintains an open-chromatin configuration in the XAF1 promoter, as assessed by the presence of both active HPMs (Fig. 5c) and de-methylated CpG dinucleotides (Fig. 5b), allowing high transcriptional responsiveness to activators (Figs 1 and 6c). Second, the loss of CTCF regulation in the XAF1 promoter, explained by the fact that CTCF interaction with its cognate binding site in the $X A F 1$ promoter is methylation sensitive (Fig. 2b,c), induces polarization from active to repressive HPMs (Fig. 5c) and accelerates CpG-dinucleotide methylation (Fig. 5b). This closed chromatin state represses transcriptional activation (Fig. 6c) and possibly contributes to apoptotic resistance. Thus, CTCF is a determinant that confers a permissive chromatin configuration to the $X A F 1$ gene, which is critical for apoptotic program culmination.

In X-chromosomal inactivation, certain genes escape from the epigenetic silencing mechanism. CTCF mediates this escape by inhibiting the propagation both of methylation and of repressive HPMs from surrounding silent regions ${ }^{34}$. In this scenario, CTCF mediates the shift between an open and closed 


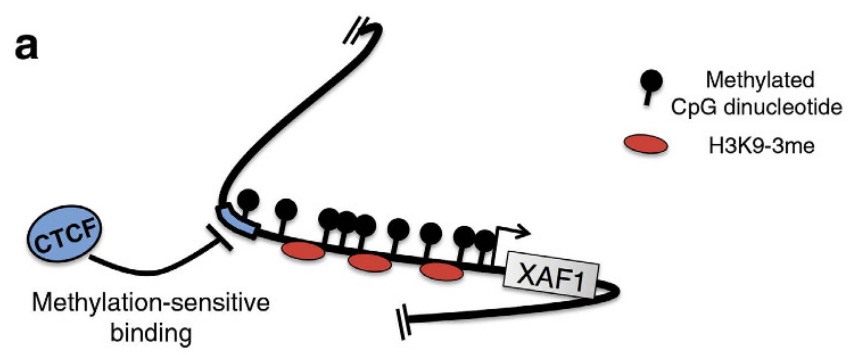

b

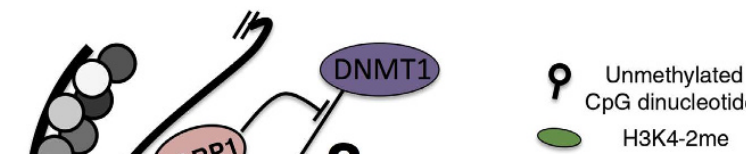

Epigenetic regulation by CTCF of the XAF1 promoter. (a) Methylation of the CTCF binding site in the XAF1 promoter, as occurs in cancer, inhibits its recognition by CTCF. This enriches repressive histone posttranslational modifications, contributing to XAF1 transcriptional silencing. (b) In demethylating conditions, CTCF is able to interact with its cognate DNA-binding site, inhibiting both CpG-dinucleotide methylation and repressive histone posttranslational modifications such as H3K9-2me. After treatment with transcriptional activators such as TNF- $\alpha$ or IFN- $\alpha$, the association between CTCF and nucleophosmin/ $\mathrm{B} 23$ is induced, allowing XAF1 promoter anchorage to the nuclear matrix and inducing transcriptional activation. Additionally, several subunits of CTCF could bridge additional genomic regions from interchromosomal or intrachromosomal locations to the same genomic anchor to which the XAF1 promoter is attached. The inhibition of CpG-dinucleotide methylation could be mediated by the inhibitory action of PARP-1 on DNMT1.

chromatin configuration by functioning as a scaffold protein to attract different enzymatic complexes involved in HPMs ${ }^{35,48,49}$. Concordantly, epigenetic silencing of CTCF-regulated genes is observed when CTCF is unable to interact with its DNA-binding site by a methylation-sensitive interaction that favours the presence of repressive, HPMs ${ }^{27,35,50}$. Importantly, reports showing the biological weight of different HPMs in the XAF1 promoter are missing. In the present report, assessing long-term transgenic behaviour allowed us to uncover the actions of CTCF on the chromatin configuration of the XAF1 promoter (Fig. 5). We observed for the first time that the absence of local CTCF in the XAF1 promoter induces the transition from active to repressive HPMs. We envision that the loss of CTCF affinity to its cognate binding-site in the XAF1 promoter could be the first driving event for the transcriptional repression of the XAF1 gene. Additional experiments are needed to support this, but it has been reported that CTCF posttranslational modifications impair its ability to interact with DNA targets ${ }^{51-54}$. Thus, lack of CTCF in its cognate site allows its methylation, impeding re-association of CTCF to it even with new posttranslational modifications arise. Consequently, this induces accelerated CpG-dinucleotide methylation and polarization from active to repressive HPM and a consequent XAF1 transcriptional silencing.

It has been described that the nuclear matrix plays an important role in the regulation of gene transcription. Chromatin is anchored by short stretches of DNA sequences called matrix attachment regions (MARs). MARs range in size from 100 to $2000 \mathrm{bp}$ and are rich in AT dinucleotide pairs and repetitive sequences. Both chromatin loop formation and the transcriptional activation of genes surrounded by MARs are dependent on nuclear matrix anchorage ${ }^{55}$. This is explainable by the fact that transcriptional factors are present in the nuclear matrix ${ }^{56}$. CTCF associates with the nuclear matrix ${ }^{57,58}$ and mediates the anchoring of DNA sequences to it, as observed in the $5^{\prime}$-HS4 chicken $\beta$-globin insulator ${ }^{58}$. In addition, the association of CTCF with the nuclear matrix depends on nucleophosmin/B23 ${ }^{59}$. However, it has been observed that IFN- $\gamma$ induces transcriptional activation of major histocompatibility complex genes, which coincides with the reorganization of chromatin loops ${ }^{60}$. Interestingly, DNA anchorage to the nuclear matrix after IFN- $\gamma$ exposure was associated with CTCF binding sites ${ }^{60}$. Therefore, CTCF reconfigures genomic regions by forming loops that affect the transcriptional gene landscape. In the present paper, we demonstrate that in cancer cells, CTCF is unable to associate with its cognate DNA-binding site in the $X A F 1$ promoter if it is methylated (Fig. 7a), thus effectively rendering it unresponsive to well-known 
activators (Fig. 7a). However, after demethylating the cognate site, CTCF is now able to associate with the XAF1 promoter to enhance transcriptional activation (Fig. 2). One intriguing possibility is that CTCF could be able to attract DNA to the nuclear matrix, mediating faster chromatin loop formation in the nuclear matrix after exposure to exogenous stimuli. Although not tested, we envision that CTCF could be able to attract the XAF1 promoter to the nuclear matrix by its association with nucleophos$\mathrm{min} / \mathrm{B} 23$, thereby inducing both chromatin loop formation and transcriptional activation of the XAF1 gene (Fig. 7b). In cancer, this putative mechanism would not occur due to the absence of CTCF in its DNA-binding site via a methylation-sensitive mechanism (Fig. 7a).

Finally, we demonstrate for the first time that CTCF is critical to maintaining key CpG-dinucleotides demethylated in the XAF1 promoter (Fig. 5b). This could be explainable by previous reports showing that CTCF associates with and activates PARP-1, which negatively regulates DNMT1, thus maintaining the CpG dinucleotides within the CTCF-binding sites free from methylation ${ }^{61,62}$. Additionally, a pool of PARP is located in the nuclear matrix and is implicated in chromatin loop formation. Although not tested, an interesting hypothesis would be the possibility that PARP-1 is a mediator of the effects of CTCF (Fig. 7b). Further experiments are required to test this.

In conclusion, we demonstrate a novel functional CTCF binding site in the XAF1 promoter. The association of CTCF with its binding site induces an open chromatin configuration by enriching active HPMs and maintaining CpG-dinucleotides free from methylation. In cancer, methylation negatively affects the interaction between CTCF and the XAF1 promoter, disabling the protective epigenetic actions of CTCF against the closed-chromatin configuration. Our finding are consistent with CTCF acting as key regulatory element in the well-accepted observation that CpG-dinucleotide methylation on the XAF1 promoter inhibits its transcriptional activation. The absence of CTCF regulation of the XAF1 gene may constitute a selective advantage during clonal evolution by means of increasing the apoptotic threshold.

\section{Methods}

Cell culture and reagents. MCF-7 (HTB22) cells were acquired from the American Type Culture Collection (ATCC) and maintained in Dulbecco's modified Eagle's medium (DMEM) supplemented with $10 \%$ fetal bovine serum (FBS). ACHN (CRL-1611) cells were acquired from the ATCC, and maintained in Eagle's Minimum Essential Medium (EMEM) supplemented with 10\% FBS. The cells were grown in a humidified incubator that was maintained at $37^{\circ} \mathrm{C}$ with $5 \% \mathrm{CO}_{2}$. Demethylating conditions were established by treating the cell lines for 3 days with $0.2 \mu \mathrm{M}$ Trichostatin-A (TSA) and $5 \mu \mathrm{M}$ 5-aza-2' -deoxycytidine (5-A-DC) (SIGMA). Daily, the medium was replaced with fresh medium containing 5-A-DC and TSA. The transfection of constructs was performed using Lipofectamine 2000 (Invitrogen). TNF- $\alpha$ and IFN- $\alpha$ were purchased from R\&D and PROSPEC, respectively.

Constructs. Genomic DNA isolated from peripheral human blood was used as a template. Primers used in this work are listed in Supplementary Table S1. Specific primers were designed to amplify by PCR the region from -1200 to $+350 \mathrm{bp}$ relative to the transcription start site (TSS) from the XAF1 gene (XAF1.2). The PCR product was purified and cloned into pTZ57r/t (Thermo). Then, XAF1.2 was subcloned into the peGFP-N1 (Clontech) expression vector to produce peGFP-N1-XAF1-promoter. Deletion of the CTCF-binding site from the peGFP-N1-XAF1-promoter plasmid was performed using the QuickChange Lightning Site-Directed Mutagenesis kit (Agilent Technologies). Following the manufacturer's protocol, we generated peGFP-N1- $\Delta$-CTCF-XAF1. Specific primers were designed to amplify by PCR the genomic region from -3000 to +350 bp relative to the transcription start site from the XAF1 gene (XAF1-promoter). The PCR product was cloned using GeneJET PCR cloning kit (Fermentas) and was then subcloned into pSEAP2-Basic (Clontech), a secreted alkaline phosphatase (SEAP) gene reporter expression vector, to produce the wild-type-XAF1-promoter-SEAP construct. Using a QuickChange Lightning Site-Directed Mutagenesis kit (Agilent Technologies), we generated the $\triangle$-CTCF-XAF1-promoter-SEAP construct, which lacked the CTCF binding site. To generate an inducible system for CTCF overexpression, CTCF was amplified from cDNA using Pfu polymerase (Stratagene) and cloned into pQCXIP (Clontech). It was then subcloned into pTRE-Tight-Bi-AcGFP1 (Clontech) to produce pTRE-Tight-Bi-AcGFP1-CTCF. All plasmids were confirmed by capillary sequencing.

Transient and stable transfection of MCF-7 cells. MCF-7 cells were seeded in 12-well plates one day before transfection. The cells were co-transfected with $0.625 \mu \mathrm{g}$ of either wild-type-XAF1-promoter-SEAP or $\triangle$-CTCF-XAF1-promoter-SEAP plasmids and $0.625 \mathrm{pg}$ of pMetLuc (Clontech), which is a plasmid encoding secreted Metridia luciferase used for transfection normalization. After $24 \mathrm{~h}$, the transfection medium was changed out for fresh medium. After $48 \mathrm{~h}$, the medium was collected to measure both SEAP and Luciferase activities using the Great EscAPe SEAP chemiluminescence kit (Clontech) and Ready-To-Glow-Secreted Luciferase Reporter Assay (Clontech), respectively.

For inducible CTCF overexpression, MCF-7 cells were transfected with $2 \mu \mathrm{g}$ pTet-On plasmid (Clontech), which encodes the rTet repressor protein. The cells were selected in G418 $(1000 \mu \mathrm{g} / \mathrm{mL})$ for 4 weeks. The pool of the resulting colonies was then expanded under G418 selection and cotransfected with $2 \mu \mathrm{g}$ pTRE-Tight-Bi-AcGFP1-CTCF with $1 \mu \mathrm{g}$ pQPCXIP empty plasmid. Stable cell clones were selected with puromycin after two weeks of selection. 
MCF-7 cells were seeded in 6-well plates. After 1 day, the cells were transfected with $2 \mu \mathrm{g}$ of either peGFP-N1-XAF1-promoter or peGFP-N1- $\Delta$-CTCF-XAF1-promoter plasmids. After $48 \mathrm{~h}$, the cells were selected with G418 (1000 $\mathrm{g} / \mathrm{mL})$ for 4 weeks. Then, G418-resistan cells were analysed by fluorescence activate cell sorting (FACS). The resistant cells were further cultured for 30 days in the absence of G418 and were analysed by FACS. Then, single cell clones were isolated. The single cell clones were continuously cultured further in the absence of G418 for 35 days, and reporter gene expression was evaluated by FACS.

Transient transfection of small interfering RNAs (siRNAs) against CTCF. ACHN and MCF-7 cells were seeded in 6-well plates. After one day, the cells were treated in demethylating conditions, as indicated above. The cells were then transfected with $0.1 \mu \mathrm{M}$ human CTCF small interfering RNAs (siRNAs; Qiagen) using Lipofectamine ${ }^{\circledR} 2000$ (Invitrogen). After $24 \mathrm{~h}$, the transfection medium was replaced with fresh medium containing demethylating agents. RNA isolation was performed $48 \mathrm{~h}$ post-transfection using TRIzol reagent (Invitrogen). RNA was converted to cDNA using random primers and SuperScript ${ }^{\circledR}$ VILO (Invitrogen).

Bisulphite DNA sequencing analysis. DNA was extracted from either MCF-7 cells or MCF-7 stable cell lines using the FlexiGene DNA Kit (Qiagen). DNA (1.5 $\mu$ g) was bisulphite converted using the Zymo EZ DNA Methylation Kit (Zymo Research) according to the manufacturer's protocol. The bisulphite-converted DNA was subjected to PCR amplification using specific primers to the XAF1 promoter (XAF-EnBis/XAF-EnBia). A nested-PCR amplification strategy was used to amplify DNA converted from stable cell lines using specific primers against the plasmid sequence (1.2GFPBis/1.2GFPBia) in the first PCR reaction to avoid amplification of the XAF1 endogenous promoter. The product from this PCR was used in a second round of PCR amplification using specific primers against the XAF1 promoter, as described above. PCR products were gel purified and cloned using the GeneJET PCR cloning kit (Fermentas), and positive clones were sent for sequencing.

Chromatin immunoprecipitation. Cells $\left(3 \times 10^{6}\right)$ were fixed with $1 \%$ formaldehyde and neutralized by adding $0.125 \mathrm{M}$ glycine. The cells were then lysed in cell lysis buffer (10 mM EDTA, $50 \mathrm{mM}$ TRIS-HCl $\mathrm{pH} 8,1 \%$ SDS, protease inhibitor cocktail). The cell lysate was sonicated to obtain soluble chromatin with a mean length of $400 \mathrm{bp}$. Chromatin immunoprecipitation (ChIP) was performed using a specific antibody raised against CTCF (C02-2899; Cell Signaling Technology) or CTCF (07-729; Millipore). Specific antibodies against H3K9me3 (ab8898 Abcam) and H3K4me2 (7766 Abcam ab) were used to perform ChIP assays on soluble chromatin from single cell clones. The DNA recovered after ChIP was subjected to PCR amplification using the following primers: XAF-CTCF-s/XAF-CTCF-as was used for the putative CTCF binding site of the XAF1 promoter; two set of primers, IGF2-CTCF-s/IGF2-CTCF-as ${ }^{63}$ and MYC-CTCF-s/MYC-CTCF-as ${ }^{64}$, were used as positive controls for the CTCF-DNA interaction; and a negative control, NEG-CTCF-s/NEG-CTCF-as, was also included.

Immunoblotting. Protein fractions were subjected to either $15 \%$ or $18 \%$ SDS-PAGE and transferred to Immobilon P membranes (Millipore). Next, the membranes were incubated with the indicated antibodies overnight, and the blots were visualized using the Immobilon Western kit (Millipore) with a peroxidase-labelled secondary antibody, according to the manufacturer's protocols.

Protein immunoprecipitation assay. The cells were washed with PBS, scraped and centrifuged at $2,000 \mathrm{rpm}$ for $3 \mathrm{~min}$. The cells were lysed using $1 \mathrm{~mL}$ of TNTE-5 buffer $(50 \mathrm{mM}$ Tris pH $7.4,150 \mathrm{mM}$ $\mathrm{NaCl}, 0.5 \%$ Triton, $1 \mathrm{mM}$ EDTA and $1 \mathrm{X}$ protease inhibitor cocktail) and incubated at $4{ }^{\circ} \mathrm{C}$ for $15 \mathrm{~min}$. The lysates were centrifuged at $14000 \mathrm{~g}$ for $10 \mathrm{~min}$. The supernatants were incubated with $10 \mu \mathrm{L}$ of recombinant protein $\mathrm{G}$ agarose beads (Life Technologies) for $1 \mathrm{~h}$. After incubation, the lysates were centrifuged at $14000 \mathrm{~g}$ for $30 \mathrm{sec}$. The supernatants were incubated overnight at $4{ }^{\circ} \mathrm{C}$ with $3 \mu \mathrm{L}$ of primary antibody with constant agitation. Next, $20 \mu \mathrm{L}$ of recombinant protein $\mathrm{G}$ agarose beads was added to each lysate, and the lysates were then incubated with constant agitation for $1 \mathrm{~h}$ on ice. The lysates were next centrifuged at $14000 \mathrm{~g}$ for $10 \mathrm{sec}$. The resulting pellets were washed twice with TNTE-1 buffer $(50 \mathrm{mM}$ Tris at $\mathrm{pH}$ 7.4, $150 \mathrm{mM} \mathrm{NaCl}, 0.1 \%$ Triton, $1 \mathrm{mM}$ EDTA and $1 \mathrm{X}$ protease inhibitor cocktail), followed by boiling in Laemmli sample solution (100 mM Tris pH 6.8, 20\% Glycerol, 2\% SDS, 0.05\% bromophenol blue and $100 \mathrm{mM}$ DTT) for further analysis.

Cell viability assay. Cell viability was measured colorimetrically using the MTS-PMS assay (CellTiter $96{ }^{\circledR}$ Aqueous Non-Radioactive Cell Proliferation Assay; Promega) according to the manufacturer's protocol. Briefly, the cells were seeded in 96 -well plates, and $24 \mathrm{~h}$ after treatment, the reagents from the kit were added to the culture medium. After $2 \mathrm{~h}$ of incubation, the absorbance was measured at a wavelength of $490 \mathrm{~nm}$ using a microplate reader.

RT-qPCR. Total RNA was extracted using TRIzol reagent (Invitrogen) according to the manufacturer's instructions. Briefly, $2 \mu \mathrm{g}$ of total RNA was used for cDNA synthesis with random hexamers. Quantitative PCR was carried out using an ABI PRISM 7900 Sequence Detection System (Applied Biosystems) using 
IDT Prime Time qPCR Primers and ZEN Double-Quenched Probe for detecting XAF1, CTCF, and HPRT genes. The TaqMan Universal PCR Master Mix (Applied Biosystems) was used. The geometric mean of housekeeping gene HPRT was used as an internal control to normalize the variability in expression levels. Results were analyzed by the comparative $2^{-\Delta \Delta \mathrm{CT}}$ method to calculate fold changes in gene expression $^{65}$. The plotted results include the mean + s.e.m. from at least three independent biological experiments.

Statistical analysis. GraphPad Prism version 5.0 for Mac Os X (La Jolla, CA) was used to perform statistical analyses. One-way analysis of variance was performed, and the Bonferroni post-test was used at $95 \%$ confidence intervals to determine significant differences.

\section{References}

1. Liston, P. et al. Identification of XAF1 as an antagonist of XIAP anti-Caspase activity. Nat Cell Biol 3, 128-133 (2001).

2. Kim, K. S., Heo, J. I., Choi, K. J. \& Bae, S. Enhancement of cellular radiation sensitivity through degradation of Chk1 by the XIAP-XAF1 complex. Cancer Biol Ther 15, 1622-1634 (2014).

3. Tse, M. K. et al. Domain organization of XAF1 and the identification and characterization of XIAP(RING) -binding domain of XAF1. Protein Sci 21, 1418-1428 (2012).

4. Arora, V. et al. Degradation of survivin by the X-linked inhibitor of apoptosis (XIAP)-XAF1 complex. J Biol Chem 282, 26202-26209 (2007).

5. Fong, W. G. et al. Expression and genetic analysis of XIAP-associated factor 1 (XAF1) in cancer cell lines. Genomics 70, 113-122 (2000).

6. Xia, Y., Novak, R., Lewis, J., Duckett, C. S. \& Phillips, A. C. Xaf1 can cooperate with TNFalpha in the induction of apoptosis, independently of interaction with XIAP. Mol Cell Biochem 286, 67-76 (2006).

7. Zou, B. et al. XIAP-associated factor 1 (XAF1), a novel target of p53, enhances p53-mediated apoptosis via post-translational modification. Mol Carcinog 51, 422-432 (2012).

8. Wang, J. et al. Identification of XAF1 as a novel cell cycle regulator through modulating G(2)/M checkpoint and interaction with checkpoint kinase 1 in gastrointestinal cancer. Carcinogenesis 30, 1507-1516 (2009).

9. Zou, B. et al. Correlation between the single-site CpG methylation and expression silencing of the XAF1 gene in human gastric and colon cancers. Gastroenterology 131, 1835-1843 (2006).

10. Wang, Y. et al. Association of expression of XIAP-associated factor 1 (XAF1) with clinicopathologic factors, overall survival, microvessel density and cisplatin-resistance in ovarian cancer. Regul Pept 178, 36-42 (2012).

11. Huang, J. et al. XAF1 as a prognostic biomarker and therapeutic target in pancreatic cancer. Cancer Sci 101, 559-567 (2010).

12. Chen, X. Y., He, Q. Y. \& Guo, M. Z. XAF1 is frequently methylated in human esophageal cancer. World J Gastroenterol 18, 2844-2849 (2012).

13. Sakemi, R. et al. X-linked inhibitor of apoptosis (XIAP) and XIAP-associated factor-1 expressions and their relationship to apoptosis in human hepatocellular carcinoma and non-cancerous liver tissues. Oncol Rep 18, 65-70 (2007).

14. Ng, K. C., Campos, E. I., Martinka, M. \& Li, G. XAF1 expression is significantly reduced in human melanoma. J Invest Dermatol 123, 1127-1134 (2004).

15. Fang, X. et al. Switch to full-length of XAF1 mRNA expression in prostate cancer cells by the DNA methylation inhibitor. Int $J$ Cancer 118, 2485-2489 (2006).

16. Kempkensteffen, C. et al. Gene expression and promoter methylation of the XIAP-associated Factor 1 in renal cell carcinomas: correlations with pathology and outcome. Cancer Lett 254, 227-235 (2007).

17. Lee, M. G. et al. Promoter CpG hypermethylation and downregulation of XAF1 expression in human urogenital malignancies: implication for attenuated p53 response to apoptotic stresses. Oncogene 25, 5807-5822 (2006).

18. Chung, S. K. et al. Frequent alteration of XAF1 in human colorectal cancers: implication for tumor cell resistance to apoptotic stresses. Gastroenterology 132, 2459-2477 (2007).

19. Lou, Y. F. et al. Combination of gefitinib and DNA methylation inhibitor decitabine exerts synergistic anti-cancer activity in colon cancer cells. PLoS One 9, e97719 (2014).

20. Micali, O. C. et al. Silencing of the XAF1 gene by promoter hypermethylation in cancer cells and reactivation to TRAILsensitization by IFN-beta. BMC Cancer 7, 52 (2007).

21. Zhu, L. M. et al. Tumor suppressor XAF1 induces apoptosis, inhibits angiogenesis and inhibits tumor growth in hepatocellular carcinoma. Oncotarget 5, 5403-5415 (2014).

22. Tu, S. P. et al. Tumor suppressor XIAP-Associated factor 1 (XAF1) cooperates with tumor necrosis factor-related apoptosisinducing ligand to suppress colon cancer growth and trigger tumor regression. Cancer 116, 1252-1263 (2010).

23. Phillips, J. E. \& Corces, V. G. CTCF: master weaver of the genome. Cell 137, 1194-1211 (2009).

24. Ong, C. T. \& Corces, V. G. CTCF: an architectural protein bridging genome topology and function. Nat Rev Genet 15, 234-246 (2014).

25. Filippova, G. N. Genetics and epigenetics of the multifunctional protein CTCF. Curr Top Dev Biol 80, 337-360 (2008),

26. Schoenherr, C. J., Levorse, J. M. \& Tilghman, S. M. CTCF maintains differential methylation at the Igf2/H19 locus. Nat Genet 33, 66-69 (2003).

27. Szabo, P. E., Tang, S. H., Silva, F. J., Tsark, W. M. \& Mann, J. R. Role of CTCF binding sites in the Igf2/H19 imprinting control region. Mol Cell Biol 24, 4791-4800 (2004).

28. Recillas-Targa, F., De La Rosa-Velazquez, I. A. \& Soto-Reyes, E. Insulation of tumor suppressor genes by the nuclear factor CTCF. Biochem Cell Biol 89, 479-488 (2011).

29. Straszewski-Chavez, S. L. et al. XAF1 mediates tumor necrosis factor-alpha-induced apoptosis and X-linked inhibitor of apoptosis cleavage by acting through the mitochondrial pathway. J Biol Chem 282, 13059-13072 (2007).

30. Wang, J. et al. All-trans retinoic acid induces XAF1 expression through an interferon regulatory factor-1 element in colon cancer. Gastroenterology 130, 747-758 (2006).

31. Sun, Y. et al. Regulation of XAF1 expression in human colon cancer cell by interferon beta: activation by the transcription regulator STAT1. Cancer Lett 260, 62-71 (2008).

32. Reu, F. J. et al. Overcoming resistance to interferon-induced apoptosis of renal carcinoma and melanoma cells by DNA demethylation. J Clin Oncol 24, 3771-3779 (2006).

33. Ziebarth, J. D., Bhattacharya, A. \& Cui, Y. CTCFBSDB 2.0: a database for CTCF-binding sites and genome organization. Nucleic Acids Res 41, D188-D194 (2013). 
34. Filippova, G. N. et al. Boundaries between chromosomal domains of X inactivation and escape bind CTCF and lack CpG methylation during early development. Dev Cell 8, 31-42 (2005).

35. Batlle-Lopez, A. et al. Novel CTCF binding at a site in exon1A of BCL6 is associated with active histone marks and a transcriptionally active locus. Oncogene 34, 246-256 (2015).

36. Witcher, M. \& Emerson, B. M. Epigenetic silencing of the p16(INK4a) tumor suppressor is associated with loss of CTCF binding and a chromatin boundary. Mol Cell 34, 271-284 (2009).

37. Peng, Z. et al. Epigenetic repression of RARRES1 is mediated by methylation of a proximal promoter and a loss of CTCF binding. PLoS One 7, e36891 (2012)

38. Joshi, M., Keith Pittman, H., Haisch, C. \& Verbanac, K. Real-time PCR to determine transgene copy number and to quantitate the biolocalization of adoptively transferred cells from EGFP-transgenic mice. Biotechniques 45, 247-258 (2008).

39. Leaman, D. W. et al. Identification of X-linked inhibitor of apoptosis-associated factor- 1 as an interferon-stimulated gene that augments TRAIL Apo2L-induced apoptosis. J Biol Chem 277, 28504-28511 (2002).

40. Leaman, D. W. et al. Novel growth and death related interferon-stimulated genes (ISGs) in melanoma: greater potency of IFNbeta compared with IFN-alpha2. J Interferon Cytokine Res 23, 745-756 (2003).

41. Plenchette, S., Cheung, H. H., Fong, W. G., LaCasse, E. C. \& Korneluk, R. G. The role of XAF1 in cancer. Curr Opin Investig Drugs 8, 469-476 (2007).

42. Egger, G., Liang, G., Aparicio, A. \& Jones, P. A. Epigenetics in human disease and prospects for epigenetic therapy. Nature 429, 457-463 (2004).

43. Feinberg, A. P., Ohlsson, R. \& Henikoff, S. The epigenetic progenitor origin of human cancer. Nat Rev Genet 7, 21-33 (2006).

44. Esteller, M. Epigenetics in cancer. N Engl J Med 358, 1148-1159 (2008).

45. Baylin, S. B. \& Herman, J. G. DNA hypermethylation in tumorigenesis: epigenetics joins genetics. Trends Genet 16, 168-174 (2000).

46. Docquier, F. et al. Heightened expression of CTCF in breast cancer cells is associated with resistance to apoptosis. Cancer Res 65, 5112-5122 (2005).

47. Mendez-Catala, C. F. et al. A novel mechanism for CTCF in the epigenetic regulation of Bax in breast cancer cells. Neoplasia 15, 898-912 (2013)

48. Li, T. et al. CTCF regulates allelic expression of Igf2 by orchestrating a promoter-polycomb repressive complex 2 intrachromosomal loop. Mol Cell Biol 28, 6473-6482 (2008).

49. Soto-Reyes, E. \& Recillas-Targa, F. Epigenetic regulation of the human p53 gene promoter by the CTCF transcription factor in transformed cell lines. Oncogene 29, 2217-2227 (2010).

50. Eldholm, V., Haugen, A. \& Zienolddiny, S. CTCF mediates the TERT enhancer-promoter interactions in lung cancer cells: identification of a novel enhancer region involved in the regulation of TERT gene. Int J Cancer 134, 2305-2313 (2014).

51. Wang, J., Wang, Y. \& Lu, L. De-SUMOylation of CCCTC binding factor (CTCF) in hypoxic stress-induced human corneal epithelial cells. J Biol Chem 287, 12469-12479 (2012).

52. MacPherson, M. J., Beatty, L. G., Zhou, W., Du, M. \& Sadowski, P. D. The CTCF insulator protein is posttranslationally modified by SUMO. Mol Cell Biol 29, 714-725 (2009).

53. Klenova, E. M. et al. Functional phosphorylation sites in the C-terminal region of the multivalent multifunctional transcriptional factor CTCF. Mol Cell Biol 21, 2221-2234 (2001).

54. Venkatraman, B. \& Klenova, E. Role of CTCF poly(ADP-Ribosyl)ation in the regulation of apoptosis in breast cancer cells. Indian J Med Paediatr Oncol 36, 49-54 (2015).

55. Ottaviani, D., Lever, E., Takousis, P. \& Sheer, D. Anchoring the genome. Genome Biol 9, 201 (2008).

56. Szentirmay, M. N. \& Sawadogo, M. Spatial organization of RNA polymerase II transcription in the nucleus. Nucleic Acids Res 28, 2019-2025 (2000).

57. Dunn, K. L., Zhao, H. \& Davie, J. R. The insulator binding protein CTCF associates with the nuclear matrix. Exp Cell Res 288, 218-223 (2003).

58. Yusufzai, T. M. \& Felsenfeld, G. The $5^{\prime}$-HS4 chicken beta-globin insulator is a CTCF-dependent nuclear matrix-associated element. Proc Natl Acad Sci USA 101, 8620-8624 (2004).

59. Yusufzai, T. M., Tagami, H., Nakatani, Y. \& Felsenfeld, G. CTCF tethers an insulator to subnuclear sites, suggesting shared insulator mechanisms across species. Mol Cell 13, 291-298 (2004).

60. Ottaviani, D. et al. CTCF binds to sites in the major histocompatibility complex that are rapidly reconfigured in response to interferon-gamma. Nucleic Acids Res 40, 5262-5270 (2012).

61. Guastafierro, T. et al. CCCTC-binding factor activates PARP-1 affecting DNA methylation machinery. J Biol Chem 283, 21873-21880 (2008).

62. Zampieri, M. et al. ADP-ribose polymers localized on Ctcf-Parp1-Dnmt1 complex prevent methylation of Ctcf target sites. Biochem J 441, 645-652 (2012).

63. Davalos-Salas, M. et al. Gain of DNA methylation is enhanced in the absence of CTCF at the human retinoblastoma gene promoter. BMC Cancer 11, 232 (2011).

64. Vetchinova, A. S., Akopov, S. B., Chernov, I. P., Nikolaev, L. G. \& Sverdlov, E. D. Two-dimensional electrophoretic mobility shift assay: identification and mapping of transcription factor CTCF target sequences within an FXYD5-COX7A1 region of human chromosome 19. Anal Biochem 354, 85-93 (2006).

65. Livak, K. J. \& Schmittgen, T. D. Analysis of relative gene expression data using real-time quantitative PCR and the 2(-Delta Delta C(T)) Method. Methods 25, 402-408 (2001).

\section{Acknowledgements}

Georgina Victoria-Acosta was a recipient of a $\mathrm{PhD}$ fellowship provided by Consejo Nacional de Ciencia y Tecnologia (CONACYT) [grant number 267949], and this study is part of her doctoral thesis from the Biomedical Sciences Doctorate Program, Universidad Nacional Autonoma de Mexico. This work was partly supported by CONACYT grant 132931 to Jorge Melendez-Zajgla. The authors thank Jose Luis Cruz-Colin for his help in cell culture techniques.

\section{Author Contributions}

G.V.-A. performed gain-of-function and loss-of-function assays, RT-PCRs, transgene assays, bisulfite genomic sequencing and analyzed data. K.V.-S. performed gene reporter assays. L.J.-H. and L.M.-G. performed western-blots and statistical analyses. V.M. interpreted data and reviewed the manuscript. G.U.M.-R. performed all plasmid constructs and ChIP assays, conceived experiments, analyzed and 
interpreted data, and wrote the manuscript. J.M.-Z. conceived experiments, analyzed and interpreted data, reviewed the manuscript and wrote the final version.

\section{Additional Information}

Supplementary information accompanies this paper at http://www.nature.com/srep

Competing financial interests: The authors declare no competing financial interests.

How to cite this article: Victoria-Acosta, G. et al. Epigenetic silencing of the XAF1 gene is mediated by the loss of CTCF binding. Sci. Rep. 5, 14838; doi: 10.1038/srep14838 (2015).

(c) (i) This work is licensed under a Creative Commons Attribution 4.0 International License. The images or other third party material in this article are included in the article's Creative Commons license, unless indicated otherwise in the credit line; if the material is not included under the Creative Commons license, users will need to obtain permission from the license holder to reproduce the material. To view a copy of this license, visit http://creativecommons.org/licenses/by/4.0/ 


\section{SCIENTIFIC REP RTS}

OPEN Corrigendum: Epigenetic silencing
of the XAF1 gene is mediated by
the loss of CTCF binding

Georgina Victoria-Acosta, Karla Vazquez-Santillan, Luis Jimenez-Hernandez, Laura MuñozGalindo, Vilma Maldonado, Gustavo Ulises Martinez-Ruiz \& Jorge Melendez-Zajgla

Scientific Reports 5:14838; doi: 10.1038/srep14838; published online 07 October 2015; updated on 08 February 2016

In the Supplementary Information file originally published with this Article, Supplementary Figures 1c and 1d were incorrectly labelled as Figure $1 \mathrm{a}$ and $1 \mathrm{~b}$. In addition, Supplementary Figures $2 \mathrm{~b}, 2 \mathrm{c}$ and $2 \mathrm{~d}$ were incorrectly labelled as Figure 1c, 1d and 1e. Lastly, Supplementary Figures 1a, 1b and 2a were omitted. These errors have been corrected in the Supplementary Information that now accompanies the Article.

(c) (i) This work is licensed under a Creative Commons Attribution 4.0 International License. The images or other third party material in this article are included in the article's Creative Commons license, unless indicated otherwise in the credit line; if the material is not included under the Creative Commons license, users will need to obtain permission from the license holder to reproduce the material. To view a copy of this license, visit http://creativecommons.org/licenses/by/4.0/ 